\title{
Exercise Stimulates the Mitogen-activated Protein Kinase Pathway in Human Skeletal Muscle
}

\author{
Doron Aronson, ${ }^{\star}$ Mariona A. Violan, ${ }^{\ddagger}$ Scott D. Dufresne, ${ }^{*}$ David Zangen, ${ }^{\star}$ Roger A. Fielding, ${ }^{\ddagger}$ and Laurie J. Goodyear* \\ *Research Division, Joslin Diabetes Center, Department of Medicine, Brigham and Women's Hospital and Harvard Medical School; and \\ the ${ }^{\ddagger}$ Department of Health Sciences, Sargent College of Allied Health Professions, Boston University, Boston, Massachusetts 02215
}

\begin{abstract}
Physical exercise can cause marked alterations in the structure and function of human skeletal muscle. However, little is known about the specific signaling molecules and pathways that enable exercise to modulate cellular processes in skeletal muscle. The mitogen-activated protein kinase (MAPK) cascade is a major signaling system by which cells transduce extracellular signals into intracellular responses. We tested the hypothesis that a single bout of exercise activates the MAPK signaling pathway. Needle biopsies of vastus lateralis muscle were taken from nine subjects at rest and after $\mathbf{6 0} \mathrm{min}$ of cycle ergometer exercise. In all subjects, exercise increased MAPK phosphorylation, and the activity of its downstream substrate, the p90 ribosomal S6 kinase 2. Furthermore, exercise increased the activities of the upstream regulators of MAPK, MAP kinase kinase, and Raf-1. When two additional subjects were studied using a onelegged exercise protocol, MAPK phosphorylation and p90 ribosomal S6 kinase 2, MAP kinase kinase 1, and Raf-1 activities were increased only in the exercising leg. These studies demonstrate that exercise activates the MAPK cascade in human skeletal muscle and that this stimulation is primarily a local, tissue-specific phenomenon, rather than a systemic response to exercise. These findings suggest that the MAPK pathway may modulate cellular processes that occur in skeletal muscle in response to exercise. (J. Clin. Invest. 1997. 99:1251-1257.) Key words: Raf-1 • mitogen-activated protein kinase kinase (MEK) • 90-kD ribosomal S6 kinase $($ RSK) • signal transduction • mechanical stress
\end{abstract}

\section{Introduction}

Increased levels of physical activity can have salutary effects on several human diseases including diabetes (1) and congestive heart failure (2). Some of these beneficial effects result from the numerous adaptations that occur in skeletal muscle in response to chronic exercise (2). Examples of these tissue-specific changes include increases in mitochondrial mass and muscle oxidative capacity $(3,4)$, a shift in skeletal myofibers to a more slow-oxidative phenotype (5-7), and increased sensitivity of muscle glucose uptake to insulin (8). Most of these functional adaptations result from the cumulative effects of re-

Address correspondence to Laurie J. Goodyear, Joslin Diabetes Center, One Joslin Place, Boston, MA 02215. Phone: 617-732-2573; FAX: 617-732-2650; E-mail: goodyeal@joslab.harvard.edu

Received for publication 21 October 1996 and accepted in revised form 7 January 1997.

J. Clin. Invest.

(C) The American Society for Clinical Investigation, Inc.

0021-9738/97/03/1251/07 \$2.00

Volume 99, Number 6, March 1997, 1251-1257 peated bouts of exercise on the expression of critical muscle proteins (e.g., citrate synthase, myosin heavy chain isoforms, GLUT-4 $(7,9,10)$. Although the increased expression of muscle proteins with exercise has long been known to result from changes in rates of transcription and translation $(7,9)$, the intracellular signaling mechanisms that link the biochemical and mechanical events of muscle contraction with these cellular processes are not known. Indeed, despite a wealth of literature characterizing the molecular details of several signaling pathways, little of this information has been incorporated into understanding the molecular basis for the clinically important adaptations that occur in skeletal muscle in response to exercise.

The mitogen-activated protein kinase $(\mathrm{MAPK})^{1}$ cascade is a ubiquitously expressed intracellular network of proteins that forms a major signaling system by which cells transduce extracellular cues into intracellular responses $(11,12)$. Numerous growth factors, hormones, and environmental stressors can stimulate this signaling cascade, with activation typically occurring via a plasma membrane receptor molecule, the adaptor proteins Shc and GRB2, and the guanine nucleotide exchange factor SOS. Stimulation of these molecules leads to Ras and Raf-1 activation $(11,12)$. Raf-1 phosphorylates the mitogenactivated protein (MAP) kinase kinase (MEK) $(13,14)$, which in turn activates the $\mathrm{p} 42^{\mathrm{MAPK}}$ and $\mathrm{p} 44^{\mathrm{MAPK}}$ isoforms of MAPK (15). MAPK, also called extracellular regulated kinases, can phosphorylate and activate various proteins in the cytoplasm as well as in the nucleus. One set of substrates is cytosolic, such as the p90 ribosomal S6 kinase (RSK) (16) and phospholipase $A_{2}(17)$, whereas a fraction of the activated MAPK population translocates into the nucleus $(18,19)$ and phosphorylates several transcription factors, including c-myc, c-fos, and Elk-1 (11, 12, 20-24). RSK itself may act as a mediator between the signal transduction pathway and intranuclear events, since it can translocate to the nucleus (19) and phosphorylate c-fos, Elk-1, and cAMP-responsive element-binding protein (CREB) $(12,24,25)$.

We hypothesized that exercise, a stimulus that plays a role in determining skeletal muscle functional capabilities, is likely to activate the MAPK signaling pathway in skeletal muscle. In the current study, we show that a single bout of exercise in human subjects leads to activation of the MAPK signaling cascade in skeletal muscle. Our data also suggest that the stimulation of this pathway is restricted to the exercising muscle, rather than a systemic response to exercise.

\section{Methods}

\section{Subjects}

15 young healthy volunteers (11 males and 4 females) were screened by a health history questionnaire and physical examination. Exclu-

1. Abbreviations used in this paper: GST, glutathione S-transferase; IE, immediate early; MAP, mitogen-activated protein; MAPK, MAP kinase; MEK, MAP kinase kinase; RSK, p90 ribosomal S6 kinase 2; $\mathrm{VO}_{2 \mathrm{MAX}}$, maximal oxygen consumption. 
Table I. Subject Characteristics

\begin{tabular}{lr}
\hline Exercising subjects $(n=11)$ & \\
\hline Age & $23.7 \pm 1.6$ \\
Weight $(\mathrm{Kg})$ & $70.5 \pm 2.9$ \\
Height $(\mathrm{cm})$ & $172.9 \pm 2.8$ \\
$\mathrm{VO}_{2 \mathrm{MAX}}(\mathrm{ml} / \mathrm{kg} \cdot \min )$ & $45.9 \pm 2.4$
\end{tabular}

Values are mean \pm SEM

sion criteria included any clinical evidence for cardiac, pulmonary, or hematologic abnormalities. To determine the appropriate intensity for the acute bout of exercise, subjects underwent maximal oxygen consumption $\left(\mathrm{VO}_{2 \mathrm{MAX}}\right)$ testing several days before participation in the study protocol. The physical characteristics of the subjects are presented in Table I.

\section{Experimental protocols}

The study was approved by the Human Research Committees of the Joslin Diabetes Center and Boston University. The procedures involved in the study were fully described, and informed consent was obtained from each subject. The study consisted of three experimental protocols (Fig. 1).

Protocol 1. Four subjects were studied at rest and after $60 \mathrm{~min}$ of cycle ergometer exercise at a load corresponding to $70 \%$ of their $\mathrm{VO}_{2 \mathrm{MAX}}$. This level of exercise is associated with a significant metabolic stress even in trained individuals (26). Each subject was instructed not to perform any exercise for $72 \mathrm{~h}$ before the study and to report to the laboratory on the morning after an overnight fast. A needle biopsy (see below) of the left vastus lateralis muscle was obtained $\sim 5 \mathrm{~min}$ before the initiation of exercise. A second biopsy was taken from the right vastus lateralis muscle $\sim 3-4$ min after completion of 60 $\mathrm{min}$ of exercise. Two additional subjects served as controls, having the second muscle biopsy obtained after a 60 -min period of rest.

Protocol 2. A percutaneous needle biopsy of vastus lateralis muscle using the Bergström needle has been shown to significantly reduce insulin-stimulated glucose uptake (27). Although the mechanism of this phenomenon is not known, the effect of the biopsy procedure itself may also influence several intracellular signaling pathways (Aronson, D., R.A. Fielding, and L.J. Goodyear, unpublished observations). To exclude the possibility that a biopsy procedure per se affects the activity of the MAPK cascade, five additional subjects were studied using a modified 2-d protocol (Fig. 1). For these subjects, a basal bi- opsy was obtained from the left vastus lateralis muscle on the first day. $24 \mathrm{~h}$ later, subjects exercised for $60 \mathrm{~min}$ on a cycle ergometer at $70 \% \mathrm{VO}_{2 \mathrm{MAX}}$, followed by a second biopsy obtained from the right vastus lateralis. In two control subjects, two muscle biopsies were performed at a 24-h interval with no intervening exercise.

Protocol 3. To determine whether the observed effects of exercise on the MAPK signaling pathway occur as a result of the systemic release of hormones or cytokines during exercise, or are elicited by a local phenomenon restricted to the exercising muscles, we studied two subjects using a one-legged exercise protocol (Fig. 1). This protocol was similar to protocol 2 except that subjects exercised with one leg (right) at a relative work intensity of $70 \%$ of the one-legged $\mathrm{VO}_{2 \mathrm{MAX}}$. At the end of the exercise bout, biopsies of vastus lateralis muscle were taken from both the exercising and nonexercising leg.

\section{Muscle biopsy procedure}

Samples of vastus lateralis muscle were obtained using the percutaneous needle biopsy technique with a 5-mm diameter side-cutting Bergström needle with applied suction (28). The biopsy needle was inserted through an incision made in the skin using a local anesthetic ( $1 \%$ lidocaine hydrochloride) and sterile conditions. The incision, 3 $\mathrm{mm}$ long and $10 \mathrm{~mm}$ deep, penetrated the underlying fascia and allowed easy entry of the biopsy needle to the desired depth of $3 \mathrm{~cm}$. Once the muscle tissue was removed, it was quickly dissected free from the surrounding fat and connective tissue, divided into two separate pieces, and immediately placed in liquid nitrogen and stored at $-80^{\circ} \mathrm{C}$. One piece of tissue ( $70-130 \mathrm{mg}$ ) was processed and used for enzyme activity assays and immunoblotting. The other piece of tissue ( $\sim 20 \mathrm{mg}$ ) was used to determine glycogen concentrations.

\section{Muscle processing}

Muscle biopsies were homogenized (Polytron; Brinkman Instruments, Inc., Westbury, NY) in ice-cold lysis buffer containing $20 \mathrm{mM}$ Hepes, pH 7.4, $2 \mathrm{mM}$ EGTA, $50 \mathrm{mM} \beta$-glycerophosphate, $1 \mathrm{mM}$ DTT, $1 \mathrm{mM} \mathrm{Na} \mathrm{VO}_{4}, 1 \%$ (vol/vol) Triton X-100, $10 \%$ (vol/vol) glycerol, $10 \mu \mathrm{M}$ leupeptin, $3 \mathrm{mM}$ benzamidine, $5 \mu \mathrm{M}$ pepstatin $\mathrm{A}, 10 \mu \mathrm{g} /$ $\mathrm{ml}$ aprotinin, $1 \mathrm{mM}$ PMSF. Homogenates were rotated for $1 \mathrm{~h}$ at $4^{\circ} \mathrm{C}$, and centrifuged at $14,000 \mathrm{~g}$ for $68 \mathrm{~min}$ at $4^{\circ} \mathrm{C}$ to remove insoluble matter. Protein concentrations in crude homogenates were estimated by the Bradford method (29).

\section{Immunoblotting}

Muscle proteins (100-200 $\mu \mathrm{g})$ were solubilized in Laemmli sample buffer (50 mM Tris-HCl, pH 6.8, 2\% SDS, 10\% glycerol) and boiled for $5 \mathrm{~min}$. The samples were then resolved on an 8\% SDS-polyacrylamide gel, transferred to nitrocellulose paper, and immunoblotted

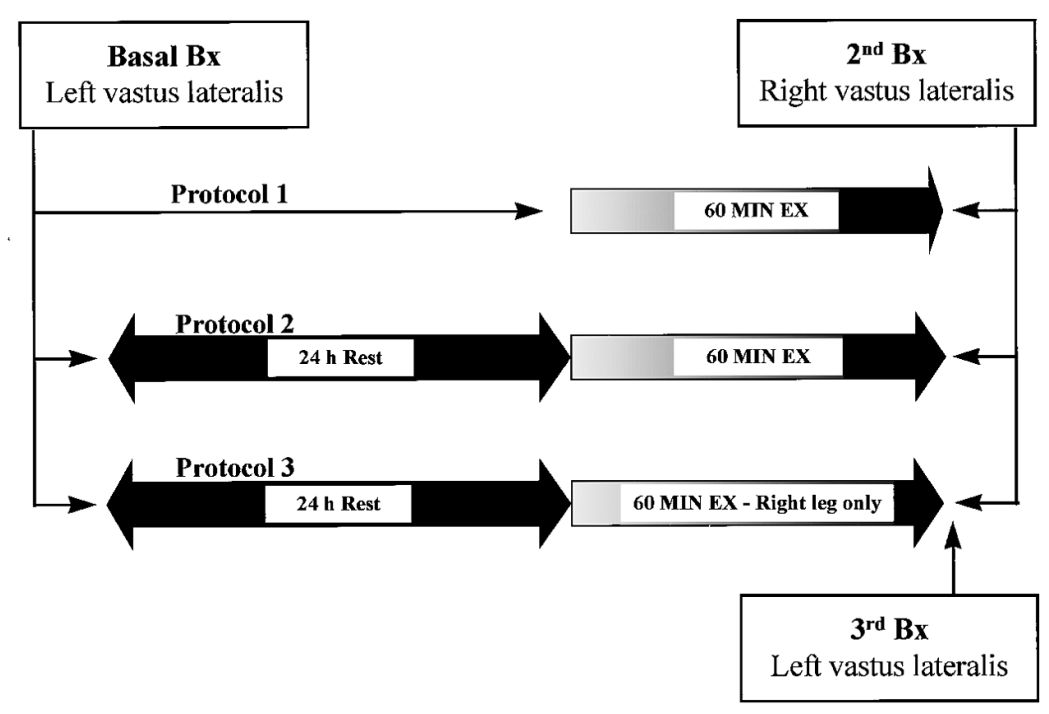

Figure 1. Study protocols. 
with anti-RSK2 $(1 \mu \mathrm{g} / \mathrm{ml})$, anti-Raf-1 $(1 \mu \mathrm{g} / \mathrm{ml})$, or a phospho-specific anti-p42 ${ }^{\mathrm{MAPK}} / \mathrm{p} 44^{\mathrm{MAPK}}(1: 2,000)$. The phospho-specific antibody recognizes the $\mathrm{p} 42^{\mathrm{MAPK}}$ and $\mathrm{p} 44^{\mathrm{MAPK}}$ isoforms of MAPK only when phosphorylated at Thr183 and Tyr185, which are required for full enzymatic activity $(15,30)$. After secondary incubation with horseradish peroxidase-conjugated anti-rabbit antibodies $(1: 2,500)$, immunoreactive proteins were detected using enhanced chemiluminescence.

\section{Kinase assays}

RSK activity was measured by an immune complex kinase assay using the 3R S6 peptide (RRLSSLRA) as substrate (31). Aliquots of $400 \mu \mathrm{g}$ muscle protein were precleared with protein A-agarose beads and then incubated with $2.5 \mu \mathrm{g}$ of polyclonal anti-RSK2 antibody for $1 \mathrm{~h}$ at $4^{\circ} \mathrm{C}$, followed by incubation with $50 \mu \mathrm{l}$ of protein A-agarose beads for an additional $2 \mathrm{~h}$. The immune complexes were washed three times with lysis buffer, three times with $\mathrm{LiCl}$ buffer $(500 \mathrm{mM}$ $\mathrm{LiCl}, 100 \mathrm{mM}$ Tris-HCl, pH 7.6, and 0.1\% Triton X-100, 1 mM DTT), and three times with RSK kinase buffer (30 mM Tris, $\mathrm{pH}$ 7.4, $10 \mathrm{mM}$ $\mathrm{MgCl}_{2}, 0.1 \mathrm{mM}$ EGTA, $1 \mathrm{mM}$ DTT). The immune complexes were resuspended in $50 \mu \mathrm{l}$ RSK kinase buffer containing $50 \mu \mathrm{g}$ S6 peptide (final concentration $0.2 \mu \mathrm{g} / \mu \mathrm{l}$ ), $40 \mu \mathrm{M}$ ATP, and $10 \mu \mathrm{Ci}\left[\gamma^{-32} \mathrm{P}\right] \mathrm{ATP}$ $(3,000 \mathrm{Ci} / \mathrm{mmol})$. Reactions were carried out at $30^{\circ} \mathrm{C}$ for $15 \mathrm{~min}$, and terminated by adding $10 \mu \mathrm{l}$ of stopping solution containing $0.6 \% \mathrm{HCl}$, $1 \mathrm{mM}$ ATP, and $1 \%$ BSA. After centrifugation, aliquots of the supernatants $(20 \mu \mathrm{l})$ were spotted onto P81 phosphocellulose papers in duplicate. The papers were washed five times with $175 \mathrm{mM}$ phosphoric acid for at least 20 min each and counted by the Cerenkov method.

MEK1 and Raf-1 activities were assayed as previously described, with several modifications (32). Muscle proteins $(250 \mu \mathrm{g})$ were incubated with $1.25 \mu \mathrm{g}$ anti-MEK1 or anti-Raf- $1 \mathrm{mAbs}$ at $4^{\circ} \mathrm{C}$ for $2 \mathrm{~h}$, followed by incubation with $40 \mu \mathrm{l}$ of protein G-agarose beads. For the MEK1 assay, immunoprecipitates were washed three times in buffer A $\left(150 \mathrm{mM} \mathrm{NaCl}, 20 \mathrm{mM}\right.$ Tris, $\mathrm{pH}$ 8.0, $10 \mathrm{mM} \mathrm{NaF}, 100 \mu \mathrm{M} \mathrm{Na}_{3} \mathrm{VO}_{4}$, $10 \%$ glycerol, $1 \%$ NP- $40,2 \mu \mathrm{g} / \mathrm{ml}$ leupeptin), and three times with MEK1 kinase buffer (25 mM Hepes, $\mathrm{pH} 7.5,10 \mathrm{mM} \mathrm{MgCl}, 2 \mathrm{mM}$ DTT). The immune complexes were resuspended in $80 \mu \mathrm{l}$ MEK1 kinase buffer containing $50 \mu \mathrm{M}$ ATP, $10 \mu \mathrm{Ci}$ of $\left[\gamma^{\left.-{ }^{32} \mathrm{P}\right] \mathrm{ATP}}(3,000 \mathrm{cpm} /\right.$ pmol), and a recombinant kinase-inactive human glutathione S-transferase (GST)-MAPK (1 $\mu \mathrm{g} /$ tube) as substrate. For the Raf-1 assay, immunoprecipitates were washed three times in buffer B $(150 \mathrm{mM}$ $\mathrm{NaCl}, 20 \mathrm{mM}$ Tris, pH 8.0, $2 \mathrm{mM}$ EDTA, $1 \mathrm{mM}$ PMSF, $100 \mu \mathrm{M}$ $\mathrm{Na}_{3} \mathrm{VO}_{4}, 10 \mu \mathrm{g} / \mathrm{ml}$ aprotinin, $10 \%$ glycerol, $1 \% \mathrm{NP}-40,2 \mu \mathrm{g} / \mathrm{ml}$ leupeptin), and three times with Raf-1 kinase buffer (30 mM Hepes, $\mathrm{pH}$ 7.4, $10 \mathrm{mM} \mathrm{MnCl} 2,5 \mathrm{mM} \mathrm{MgCl} 2,100 \mu \mathrm{M} \mathrm{Na}_{3} \mathrm{VO}_{4}, 25 \mathrm{mM} \beta$-glycerophosphate, $1 \mathrm{mM}$ DTT, and Brij $350.003 \%$ ). The Raf-1 immune complexes were resuspended in $50 \mu$ l Raf- 1 kinase buffer containing 20 $\mu \mathrm{M}$ ATP, $10 \mu \mathrm{Ci}$ of $\left[\gamma^{-32} \mathrm{P}\right] \mathrm{ATP}$, and $1 \mu \mathrm{l}$ recombinant kinase-inactive GST-MEK1 as substrate. For both assays, reactions were incubated for $30 \mathrm{~min}$ at $30^{\circ} \mathrm{C}$ and were terminated by adding Laemmli sample buffer. Products were boiled for $5 \mathrm{~min}$ and resolved on $10 \%$ SDS-PAGE. Gels were dried and the phosphorylated GST-MAPK (70 kD) or GST-MEK1 (71 kD) were quantitated by PhosphorImager and ImageQuant software (Molecular Dynamics, Inc., Sunnyvale, CA).

\section{Materials}

$\left[\gamma^{-}{ }^{32} \mathrm{P}\right]$-ATP was purchased from Du Pont-New England Nuclear (Boston, MA); anti-RSK2 (06-300) and 3R S6 RSK substrate peptide, GST-MAPK, and GST-MEK1 from Upstate Biotechnology, Inc. (Lake Placid, NY); anti-Raf-1 from Santa Cruz Biotechnology Inc. (Santa Cruz, CA); anti-MEK1 mAb and enhanced chemiluminescence kits from Transduction Laboratories (Lexington, KY); phospho-specific anti-p $42^{\mathrm{MAPK}} / \mathrm{p} 44^{\mathrm{MAPK}}$ from Quality Controlled Biochemicals Inc., Hopkinton, MA; protein A-agarose and protein G-agarose from Pierce Chemical Co. (Rockford, IL). Protein concentrations were determined using a dye reagent from Bio-Rad Laboratories (Hercules, CA), and all other chemicals were purchased from Sigma Chemical Co. (St. Louis, MO).

\section{Statistical analysis}

All data are expressed as mean \pm SEM. Differences between basal and postexercise points were determined using a paired Student's $t$ test. $P<0.05$ was considered statistically significant.

\section{Results}

Exercise-stimulated phosphorylation of the p42 and p44 MAP kinase proteins. Fully active MAPK requires phosphorylation of both threonine 183 and tyrosine $185(15,30)$, and inactivation can occur by the dephosphorylation of either residue (16). To determine the phosphorylation state of the MAPK isoforms in skeletal muscle, we used a phospho-specific antibody that recognizes only the dual-phosphorylated $42-\mathrm{kD}$ and $44-\mathrm{kD}$ proteins. This antibody is an especially valuable tool for studies of human skeletal muscle, where the small samples provide little protein for kinase activity assays. The representative immunoblots in Fig. 2 show that this antibody recognizes two proteins corresponding to the 42- and 44-kD MAPK species in human skeletal muscle.

To determine if exercise activates the MAPK signaling cascade, we first obtained muscle samples from four subjects immediately before and after $60 \mathrm{~min}$ of exercise (protocol 1). The representative immunoblot in Fig. $2 A$ shows that exercise increased the phosphorylation of both the p42 $2^{\mathrm{MAPK}}$ and $\mathrm{p} 44^{\mathrm{MAPK}}$ isoforms. Densitometric analysis of the combined $\mathrm{p} 42^{\mathrm{MAPK}}$ and p44 ${ }^{\mathrm{MAPK}}$ bands revealed that exercise increased phosphorylation in all subjects, and that the magnitude of change in phosphorylation with exercise ranged from 4-24-fold above baseline.

To insure that the stimulation of MAPK phosphorylation in exercising subjects was not affected by the baseline biopsy procedure, five additional subjects were studied with a 24-h interval between the pre- and postexercise muscle biopsies (pro-

A

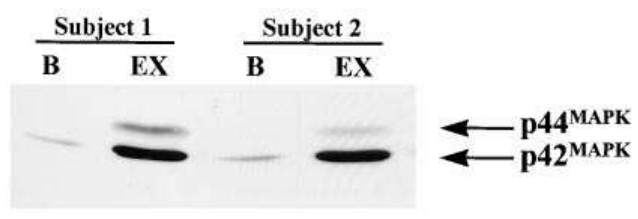

B

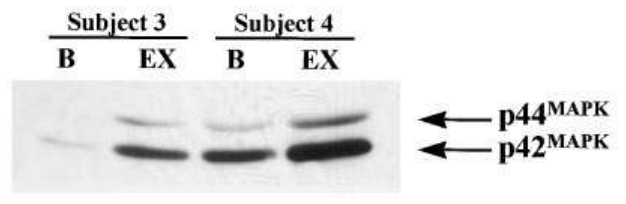

C

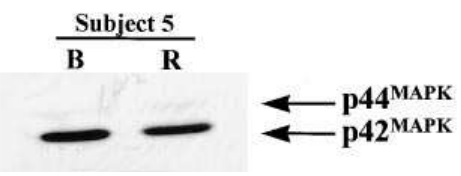

Figure 2. Exercise increases MAPK phosphorylation. Muscle proteins were subjected to Western blot analysis, and the phosphorylation of the $\mathrm{p} 42^{\mathrm{MAPK}}$ and $\mathrm{p} 44^{\mathrm{MAPK}}$ isoforms was demonstrated using specific antibodies that recognize only the dual-phosphorylated MAPK as described in Methods. $(A)$ Representative immunoblot of two subjects that participated in protocol 1. (B) Representative immunoblot of two subjects that participated in protocol 2. (C) For subject 5 , the second muscle biopsy was taken after $60 \mathrm{~min}$ of rest. Arrows indicate the $\mathrm{p} 42^{\mathrm{MAPK}}$ and $\mathrm{p} 44^{\mathrm{MAPK}}$ isoforms. $B$, baseline; $E X$, postexercise; $R$, rest. 
tocol 2). As with subjects studied in protocol 1, exercise increased MAPK dual-phosphorylation in all subjects regardless of the basal state of phosphorylation (Fig. $2 B$ ). The increase in MAPK phosphorylation ranged from 2-23-fold above basal.

In contrast to studies of cultured cells under serum-free conditions where the basal activity of the MAPK cascade is very low, the activity of the MAPK cascade in human skeletal muscle could be influenced by the many humoral factors (e.g., insulin). Thus, in vivo, large hour-to-hour or day-to-day fluctuations could obscure the ability to measure an exercise-specific activation of the MAPK pathway. Therefore we studied two control subjects for each protocol. As demonstrated in the representative immunoblot in Fig. $2 C$, there were no significant changes in MAPK phosphorylation in the control subjects.

Exercise increases RSK activity. RSK is a cytosolic kinase that can be phosphorylated and activated by MAPK (16), and may act as a mediator between signal transduction pathways and intranuclear events $(12,24,25)$. To determine if this downstream signaling element is activated in response to exercise, we measured RSK activity using an immune complex kinase assay, and in separate experiments assessed RSK phosphorylation by changes in the electrophoretic mobility of the protein using SDS-PAGE. When the basal and postexercise muscle biopsies were done on the same day (protocol 1), exercise increased RSK activity in all subjects, with the magnitude of stimulation ranging from 1.6-4.6-fold above basal (2.1 \pm 0.6 ; mean \pm SEM, $P<0.05$ ) (Fig. $3 A$, left). When the basal muscle sample was obtained $24 \mathrm{~h}$ before the exercise (protocol 2), increased RSK activity was also observed in all exercising subjects, with the degree of stimulation ranging from 2.0-2.5-fold increase above basal $(2.2 \pm 0.1$; mean \pm SEM, $P<0.05)$ (Fig. 3 $A$, right). Basal RSK activities were variable among subjects, similar to the variability observed in the basal state of MAPK phosphorylation (Fig. 2). In fact, in one subject basal RSK activity was considerably higher than all other subjects (Fig. $3 A$, inset). The variability among subjects in MAPK phosphorylation and RSK activity may reflect heterogeneity in the level of humoral or other factors that influence the activity of the MAPK pathway in human skeletal muscle. However, regard- less of the level of basal MAPK phosphorylation or RSK activity, exercise increased MAPK phosphorylation and RSK activity in all subjects. In the control subjects, RSK activity measured after $60 \mathrm{~min}$ of rest ranged from $0.8-1.4$-fold of basal, demonstrating that the hour-to-hour or day-to-day variations in RSK activities were below the increases induced by exercise. Fig. $3 B$ shows the effects of exercise on RSK phosphorylation as assessed by gel mobility shift. Both exercise protocols resulted in a decrease in the electrophoretic mobility of the RSK protein, suggesting increased phosphorylation and confirming the increase in RSK activity with exercise.

Exercise-induced stimulation of MEK1 and Raf-1 activity. In many cell types, the activation of MAPK has been shown to occur in response to the sequential phosphorylation and activation of Raf-1 and MEK $(13,15)$. However, under certain conditions MAPK and RSK activities may not correlate with their upstream activator MEK due to changes in phosphatase activities (33). Therefore, we determined if exercise stimulates MEK and Raf-1 activities in response to exercise. Of the six MEK isoforms expressed in skeletal muscle (34), MEK1 and MEK2 have been shown to phosphorylate and activate p42 $2^{\mathrm{MAPK}}$ and $\mathrm{p} 44^{\mathrm{MAPK}}$ (35). MEK 1 activity was assayed in the five subjects who participated in protocol 2 by measuring the ability of MEK1 immune complexes to phosphorylate the recombinant kinase-inactive MAPK (p44 ${ }^{\mathrm{MAPK}}$ ) (Fig. $4 A$ ). Exercise increased MEK1 activity in all exercising subjects (Fig 4B).

The serine/threonine protein kinase Raf-1 phosphorylates and activates MEK1 $(13,14,36)$. Raf- 1 is activated by phosphorylation, which is accompanied by a decrease in its electrophoretic mobility with SDS-PAGE (36). Exercise decreased the electrophoretic mobility of the Raf- 1 protein in all exercising subjects, suggesting increased phosphorylation (Fig. $5 A$ ). However, since some studies have demonstrated that Raf-1 activity does not always correlate with its level of phosphorylation $(37,38)$, we also examined the effects of exercise on Raf-1 activity towards its downstream substrate MEK1. Raf-1 activity was measured in anti-Raf-1 immune complexes using a recombinant kinase-inactive MEK1 as substrate. Exercise increased Raf-1 activity in all exercising subjects (Fig. $5 \mathrm{C}$ ).
$\mathbf{A}$

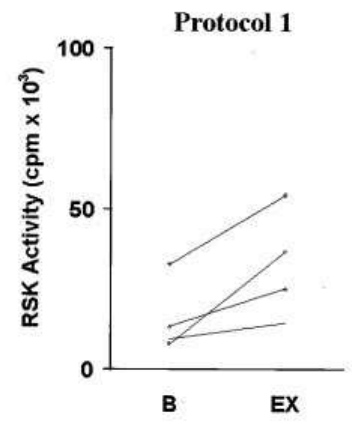

B

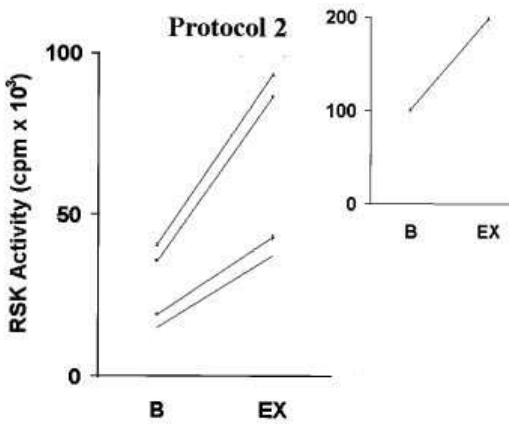

Subject 1 Subject 2 Subject 3

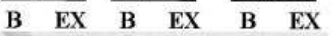

Protocol 1

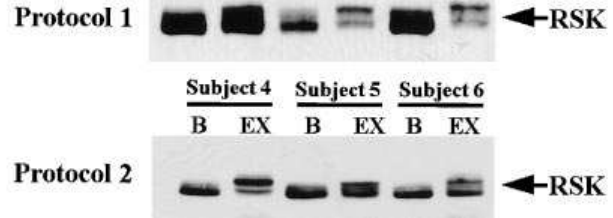

Figure 3. Effects of an acute bout of exercise on RSK activity. ( $A$ ) Muscle proteins were immunoprecipitated with $\alpha$ RSK, and RSK activity was measured in the immunoprecipitates using the 3R S6 peptide as substrate. The data are expressed as counts per min of ${ }^{32} \mathrm{P}$ incorporated into the substrate. RSK activity increased in all exercised subjects in both protocol 1 and protocol 2. Each line represents an individual subject. In the inset to protocol 2, the response of one subject with a high basal RSK activity is shown. (B) Muscle proteins were resolved on an $8 \%$ SDS-polyacrylamide gel, transferred to nitrocellulose paper, and immunoblotted with antiRSK. RSK phosphorylation in the postexercise samples was demonstrated by the decrease in the electrophoretic mobility of the RSK protein. $B$, baseline; $E X$, postexercise. 


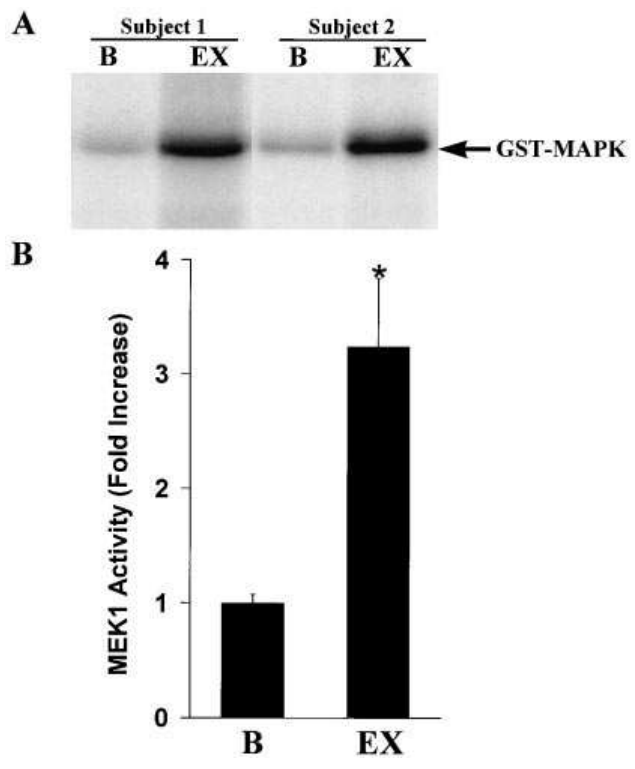

Figure 4. MEK1 activation by exercise. MEK1 protein was immunoprecipitated from muscle proteins and kinase activity was measured based on phosphorylation of GST-MAPK as described in Methods. (A) A representative autoradiogram of the $70-\mathrm{kD}$ band corresponding to the phosphorylated GST-MAPK in two subjects who underwent protocol 2. $(B)$ The intensity of phosphorylation of kinase-inactive GST-MAPK was quantitated by PhosphorImager to determine relative MEK1 activity. Data are the mean \pm SEM for the five subjects who participated in protocol 2 . Asterisk indicates significant difference from baseline $(P<0.05)$. $B$, baseline; $E X$, postexercise.

MEK1 and Raf-1 activities were not altered in muscle from nonexercising control subjects (data not shown). Taken together, these experiments suggest that the exercise-induced activation of the MAPK signaling cascade propagates from Raf-1 to RSK, in an analogous manner to that mediated by cell-surface receptors.

Activation of the MAPK signaling pathway occurs predominantly in exercising muscle. Physical activity can cause an endocrine stress response that involves a surge like secretion of catecholamines, growth hormone, and cortisol $(39,40)$. Thus, it is possible that these hormones or other systemic factors may directly or indirectly play a role in the stimulation of the MAPK pathway. Alternatively, since adaptive changes in skeletal muscle are largely confined to the exercised tissue and frequently do not require hormonal support (9), the stimulation of the MAPK signaling pathway by exercise may be a tissuespecific phenomenon. To determine if the exercise-induced stimulation of the MAPK signaling pathway occurs as a result of a local mechanism or as a systemic response to hormones or other factors, we used a one-legged exercise protocol that allowed for matched comparisons within the same subject and controlled for humoral influences and biological heterogeneity between subjects.

For the two subjects that participated in protocol 3, a basal biopsy was obtained, and $24 \mathrm{~h}$ later the subjects performed 60 min of one-legged exercise. Immediately after the exercise, one muscle sample was biopsied from the exercised leg and another sample biopsied from the nonexercised leg. There was clear stimulation of all components of the MAPK cascade only

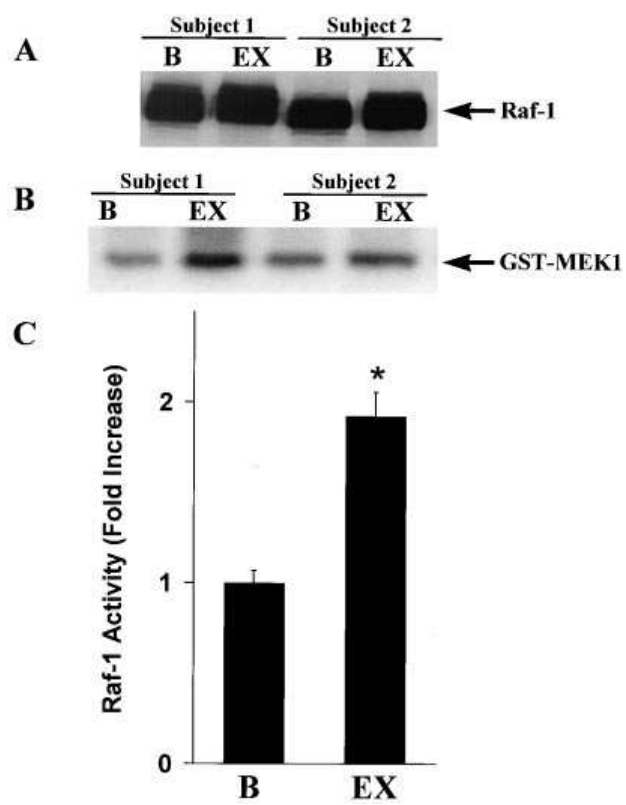

Figure 5. Activation of Raf-1 by exercise. $(A)$ Muscle proteins were electrophoresed on an $8 \%$ polyacrylamide gel and transferred to nitrocellulose filters. Protein blots were probed with anti-Raf- 1 antibody, and the bands were visualized by enhanced chemiluminescence. Exercise resulted in the phosphorylation of Raf- 1 as indicated by decreased electrophoretic mobility of the Raf-1 protein. (B) Raf-1 was immunoprecipitated from muscle proteins and assayed for its ability to phosphorylate kinase-inactive GST-MEK1 as described in Methods. The representative autoradiogram shows the $71-\mathrm{kD}$ band corresponding to the phosphorylated GST-MEK1 in two subjects who participated in protocol 2. (C) Phosphorylation of GST-MEK1 was quantified by PhosphorImager in the five subjects who participated in protocol 2. Data are mean \pm SEM. Asterisk indicates significant difference from baseline $(P<0.05)$. $B$, baseline; $E X$, postexercise.

in the exercised leg (Fig. 6). It is also important to point out that the exercise-induced stimulation of enzyme activities in the exercising leg of these subjects were of the same magnitude as that observed in subjects who exercised with both legs. These results suggest that activation of the MAPK signaling cascade occurs primarily as a local response to exercise rather than as a systemic response.

\section{Discussion}

Skeletal muscle is a highly adaptive tissue that responds to increased work with a host of metabolic and phenotypical changes. With repeated bouts of exercise, structural remodeling of skeletal muscle can occur (7), leading to enhanced functional capacity. A central issue in understanding the biological effects of exercise is to elucidate the intracellular signaling mechanisms that enable muscle cells to decipher and respond to the contractile stimulus with increased gene transcription and protein synthesis (5). Since changes in the functional properties of skeletal muscle result from the cumulative effects of repeated bouts of exercise, the initial signaling events that result in long-term adaptations should occur with each individual exercise bout. 


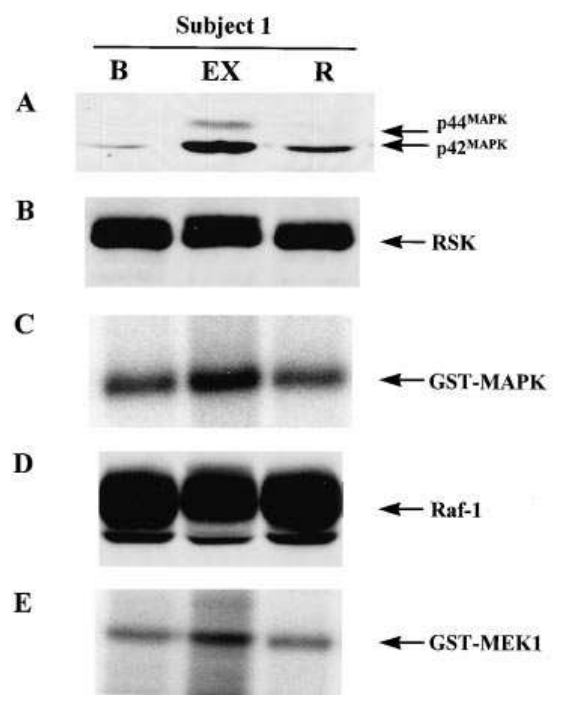

Figure 6. Activation of the MAPK pathway occurs in exercising muscle. Response of components of the MAPK cascade in a subject who participated in the one-legged exercise protocol. $(A)$ MAPK phosphorylation was increased in the postexercise sample compared with the basal state, and the nonexercising leg $(R)$. $(B)$ The decrease in the electrophoretic mobility of the RSK protein occurred only in the exercising leg. (C) MEK1 activity increased in the exercising leg. $(D)$ The decrease in the electrophoretic mobility of the Raf-1 protein occurred only in the exercising leg. $(E)$ Increased Raf-1 activity occurred only in the exercising leg. $B$, baseline; $E X$, exercising leg; $R$, resting leg.

The data from the current study demonstrate that the MAPK signaling cascade, from Raf-1 to RSK, is activated in response to a single bout of exercise in human skeletal muscle. Exercise was shown to increase Raf-1 activity and phosphorylation, increase MEK1 activity, increase $\mathrm{p} 42^{\mathrm{MAPK}}$ and $\mathrm{p} 44^{\mathrm{MAPK}}$ phosphorylation, and increase RSK activity and phosphorylation. These changes occurred in every exercising subject, and were absent in control subjects. We also demonstrate for the first time that both Raf-1 and MEK1 are activated by exercise. The effects of exercise on the MAPK signaling cascade are consistent with our previous finding showing that treadmill exercise increases MAPK phosphorylation and RSK activity in rat skeletal muscle (31). The current study also makes the important observation that the activation of the MAPK pathway is restricted to the exercising muscle. This result suggests that the stimulation of this signaling cascade is primarily a function of a local, muscle-specific mechanism, rather than a systemic response to exercise.

Several studies have demonstrated that immediate early (IE) genes are likely to be important third messengers in mechanically stimulated skeletal muscle $(9,41)$. The basal expression of IE genes is low in postmitotic striated muscle, but can be rapidly induced within hours in skeletal muscle subjected to conditions known to induce adaptive changes $(9,41)$. Michel et al. (9) have shown that neural stimulation of skeletal muscle induces an increase of IE genes products c-fos, c-jun, and egr-1, which can be detected several hours after contractile activity commences. The intracellular signaling mechanism(s) that leads to the induction of IE genes with contractile activity is not known. However, in other cell types IE genes are among the major nuclear targets of MAPK $(11,20,21,23,42)$ and RSK $(12,24,25,42)$. Thus, the current findings, taken together with previous studies, suggest that the MAPK pathway may act as a second messenger and IE gene products serve as third messengers that direct the subsequent adaptive changes that occur in skeletal muscle with repeated bouts of exercise.

How does physical exercise cause the activation of the MAPK pathway? One possibility is that the activation of the MAPK pathway during exercise is primarily mediated by hormones or cytokines released systemically. However, the onelegged exercise protocol demonstrated a predominant increase in MAPK phosphorylation and in RSK, MEK1, and Raf-1 activities in the exercising leg (Fig. 6). These findings suggest an intrinsic, contraction-induced mechanism for the activation of the MAPK pathway. Local activation of muscle MAPK signaling is fundamental to the hypothesis that this pathway is involved in converting mechanical stimuli into transcriptional responses, since increased gene expression is restricted to the contracting skeletal muscle $(9,41)$. Our data do not allow us to exclude the possibility that increased blood flow to the exercising leg, accompanied by an increased delivery of a humoral factor, is partly responsible for the predominantly unilateral activation of the MAPK pathway. However, this possibility is unlikely because in rat skeletal muscle increased contractile activity in the absence of increased blood flow potently stimulates MAPK signaling (Hayashi, T., and L.J. Goodyear, unpublished observations).

There are several potential mechanisms that could mediate the local activation of the MAPK pathway in exercising muscle. Exercise may activate intracellular signaling pathways by releasing growth factors in an autocrine fashion, which in turn stimulates their specific receptors and subsequently second messenger systems. To date, such a mechanism has been shown only in skeletal muscle cells (43) and cardiac myocytes (44) stretched in culture. Other factors that may contribute to the local stimulation of the MAPK pathway by exercise include changes in osmolarity, intracellular calcium levels, or intracellular energy depletion, all of which have been reported to activate MAPK in other cell types (45-47). Another possibility is that the extracellular matrix, which carries the external mechanical force of muscle contraction to the cell surface, can interact with cytoskeletal integrins resulting in the activation of MAPK $(48,49)$.

In summary, we have shown that activation of the MAPK pathway is a consistent response in human skeletal muscle during an acute bout of exercise, and that this response occurs predominantly in the contracting muscle. An important area of future study will be to determine if the MAPK signaling pathway serves as a link between contractile activity and transcriptional responses in human skeletal muscle.

\section{Acknowledgments}

This work was supported by National Institute of Arthritis and Musculoskeletal and Skin Diseases grant R29-AR-42238 and a grant from the Juvenile Diabetes Foundation International (L.J. Goodyear).

\section{References}

1. American Diabetes Association. 1990. Exercise and NIDDM: technical review. Diabetes Care. 13:785-789.

2. Wilson, J.R., 1995. Exercise intolerance in heart failure: importance of skeletal muscle. Circulation. 91:320-329. 
3. Baldwin, K.M., G.H. Klinkerfuss, R.T. Terjung, P.A. Molé, and J.O. Holloszy. 1972. Respiratory capacity of white, red, and intermediate muscle: adaptive response to exercise. Am. J. Physiol. 222:373-378.

4. Holloszy, J.O. 1976. Biochemical adaptations to endurance exercise in muscle. Annu. Rev. Physiol. 38:273-291.

5. Booth, F.W., and R.B. Thomason. 1991. Molecular and cellular adaptation in response to exercise: perspectives of various models. Physiol. Rev. 71: $541-585$.

6. Simoneau, J.A., M.R. Lortie, C.M. Bonlay, M.C. Marcotte, and C. Bouchard. 1985. Human skeletal muscle fiber type alteration with high-intensity intermittent training. Eur. J. Appl. Physiol. 54:250-253.

7. Pearson, A.M. 1990. Muscle growth and exercise. Crit. Rev. Food Sci. Nutr. 29:167-196.

8. Richter, E.A., L.P. Garetto, M.N. Goodman, and N.B. Ruderman. 1982. Muscle glucose metabolism in the rat: increased sensitivity to insulin. J. Clin. Invest. 69:785-793.

9. Michel, J.B., G.A. Ordway, J.A. Richardson, and R.S. Williams. 1994. Biphasic induction of immediate early gene expression accompanies activitydependent angiogenesis and myofiber remodeling of rabbit skeletal muscle. $J$. Clin. Invest. 90:277-285.

10. Ren, J.M., C.F. Semenkovich, E.A. Gulve, J. Gao, and J.O. Holloszy. 1994. Exercise induces rapid increases in GLUT4 expression, glucose transport capacity, and insulin-stimulated glycosylation storage in muscle. J. Biol. Chem. 269:14396-14401.

11. Seger, R., and E.G. Krebs. 1995. The MAPK signaling cascade. FASEB J. 9:726-735

12. Blenis, J. 1993. Signal transduction via the MAP kinases: proceed at our own RSK. Proc. Natl. Acad. Sci. USA. 90:5889-5892.

13. Lange-Carter, C.A., A.M. Pleiman, K.J. Blumer, and G.L. Johnson. 1993. A divergence in the MAP kinase regulatory network defined by MEK kinase and Raf. Science (Wash. DC). 260:315-319.

14. Yan, M., and D.M. Templeton. 1994. Identification of 2 serine residues in MEK-1 that are differentially phosphorylated during activation by Raf and MEK kinase. J. Biol. Chem. 269:19067-19073.

15. Payne, D.M., A.J. Rossamando, P. Martino, A.K. Erickson, J.-H. Her, J. Shabanowitz, D.F. Hunt, M.J. Weber, and T.W. Sturgill. 1991. Identification of the regulatory phosphorylation sites in pp42/mitogen-activated protein kinase (MAP kinase). EMBO (Eur. Mol. Biol. Organ.) J. 10:885-892.

16. Sturgill, T.W., L.B. Ray, E. Erikson, and J.L. Maller. 1988. Insulin-stimulated MAP-2 kinase phosphorylates and activates ribosomal protein S6 kinase II. Nature (Lond.). 334:715-718.

17. Long-Lih, L., M. Wartmann, A.Y. Lin, J.L. Knopf, A. Seth, and R.J. Davis. 1993. cPLA 2 is phosphorylated and activated by MAP kinase. Cell. 72 : 269-278.

18. Lenormand, P., C. Sardet, G. Pages, L'Allemain, A. Brunet, and J. Pouyssegur. 1993. Growth factors induce nuclear translocation of MAP kinase $\left(\mathrm{p} 42^{\mathrm{mapk}}\right.$ and $\left.\mathrm{p} 44^{\mathrm{mapk}}\right)$ but not of their activator MAP kinase kinase (p45 $\left.{ }^{\mathrm{mapk}}\right)$ in fibroblasts. J. Cell Biol. 122:1079-1088.

19. Chen, R.H., C. Sarnecki, and J. Blenis. 1992. Nuclear localization and regulation of Erk- and Rsk-encoded protein kinases. Mol. Cell Biol. 12:915922 .

20. Whitmarsh, A.J., P. Shore, A.D. Sharrocks, and R.J. Davis. 1995. Integration of MAP kinase signal transduction pathways at the serum response element. Science (Wash. DC). 269:403-407.

21. Hill, C.S., and R. Tresman. 1995. Transcriptional regulation of extracellular signals: mechanism and specificity. Cell. 80:199-211.

22. Hunter, T. 1995. Protein kinases and phosphatases: the yin and yang of protein phosphorylation and signaling. Cell. 80:225-236.

23. Gille, H., A.D. Sharrocks, and P.E. Shaw. 1992. Phosphorylation of transcription factor p62 $2^{\mathrm{TCF}}$ by MAP kinase stimulates ternary complex formation on $c$-fos promoter. Nature (Lond.). 358:414-417.

24. Ward, G.E., and M.W. Kirschner. 1990. Identification of cell cycle-regulated phosphorylation sites on nuclear lamin C. Cell. 61:561-577.

25. Xing, J., D.D. Ginty, and M.E. Greenberg. 1996. Coupling of the RASMAPK pathway to gene activation by RSK2, a growth factor-regulated CREB kinase. Science (Wash. DC). 273:959-963.

26. Costill, D.L., H. Thomason, and E. Roberts. 1973. Fractional utilization of the aerobic capacity during distance running. Med. Sci. Sports. 5:248-252.

27. Holck, P., N. Pørksen, M.F. Nielsen, B. Nyholm, J.F. Bal, F. Andreasen, N. Møller, and O. Schmitz. 1994. Effect of needle biopsy from the vastus lateralis muscle on insulin-stimulated glucose metabolism in humans. Am. J. Physiol. 267:E544-E548.
28. Bergström, J. 1962. Muscle electrolytes in man determined by neutron activation analysis on needle biopsy specimens. A study on normal subjects, kidney patients, and patients with chronic diarrhea. Scand. J. Clin. Lab. Invest. (Suppl.) 68:1-110

29. Bradford, M.M. 1976. A rapid and sensitive method for the quantitation of microgram quantities of protein, utilizing the principle of protein-dye binding. Anal. Biochem. 72:248-254.

30. Anderson, N.G., J.L. Maller, N.K. Tonks, and T.W. Sturgill. 1990. Requirement for integration of signals from two distinct phosphorylation pathways for activation of MAP kinase. Nature (Lond.) 343:651-653.

31. Goodyear, L.J., P.-Y. Chung, D. Sherwood, S.D. Dufresne, and D.E. Moller. 1996. Effects of exercise and insulin on mitogen-activated protein kinase signaling pathways in rat skeletal muscle. Am. J. Physiol. 271:E403-E408.

32. Alessi, D.R., P. Cohen, A. Ashworth, S. Cowlwy, S.L. Leevers, and C.J. Marshall. 1995. Assay and expression of mitogen-activated protein kinase, MAP kinase kinase, and Raf. Methods Enzymol. 225:279-300.

33. Campbell, J.S., M.-P. Wenderoth, S.D. Hauschka, and E.G. Krebs. 1995. Differential activation of mitogen-activated protein kinase in response to basic fibroblast growth factors in skeletal muscle cells. Proc. Natl. Acad. Sci. USA. 92: 870-874.

34. Han, J., J.D. Lee, Y. Jiang, Z. Li, L. Feng, and R.J. Ulevitch. 1996. Characterization of structure and function of the novel MAP kinase kinase (MKK6). J. Biol. Chem. 271:2886-2891.

35. Zheng, C.F., and K.L. Guan. 1993. Properties of MEKs, the kinases that phosphorylate and activate the extracellular signal-regulated kinases. J. Biol. Chem. 268:23933-23939.

36. Kyriakis, J.M., X. App, X. Zhang, P. Banerjee, D.L. Brautigan, U.R. Rapp, and J. Avruch. 1992. Raf-1 activates MAP kinase-kinase. Nature (Lond.). 358:417-421.

37. Samuels, M.L., M.J. Weber, J.M. Bishop, and M. McMahon. 1993. Conditional transformation of cells and rapid activation of the mitogen-activated protein kinase cascade by an estradiol-dependent Raf-1 protein kinase. Mol. Cell. Biol. 13:6241-6252.

38. Morrison, D.K., G. Heidecker, U.R. Rapp, and T.D. Copeland. 1993. Identification of the major phosphorylation sites of the Raf-1 kinase. J. Biol. Chem. 23:17309-17316.

39. Kindermann, W., Schnabel A., W.M. Schmitt, G. Biro, J. Cassens, and F. Weber. 1982. Catecholamines, growth hormone, cortisol, insulin, and sex hormones in anaerobic and aerobic exercise. Eur. J. Appl. Physiol. 49:389-399.

40. Kuoppasalmi, K., H. Haveri, S. Rehunen, M. Haufkonen, and H. Adlerkreutz. 1976. Effect of strenuous anaerobic running exercise on plasma growth hormone, cortisol, luteinizing hormone, testosterone, androstenedione, estrone and estradiol. Steroid Biochem. 7:823-829.

41. Lee, D.M., N.J. Dawes, V.M. Cox, J.E. Hesketh, and D.F. Goldspink. 1995. Immunolocalization of proto-oncogene expression in mechanically stimulated skeletal muscle. Biochem. Soc. Trans. 23:329S.

42. Chen, R.H., C. Abate, and J. Blenis. 1993. Phosphorylation of the c-fos transrepression domain by mitogen-activated protein kinase and $90-\mathrm{kDa}$ ribosomal S6 kinase. Proc. Natl. Acad. Sci. USA. 90:10952-10956.

43. Mark, S.F., and D.L. Feeback. 1996. FGF release in differentiated human skeletal muscle cultures. FASEB J. 10:502-509.

44. Sadoshima, J., Y. Xu, H.S. Slayter, and S. Izumo. 1993. Autocrine release angiotensin II mediates stretch-induced hypertrophy of cardiac myocytes in vitro. Cell. $75: 977-784$.

45. Yoshio, T., K. Tomita, M.K. Homma, H. Nonoguchi, T. Yang, T. Yamada, Y. Yuasa, E.G. Krebs, S. Sasaki, and F. Marumo. 1995. Sequential activation of Raf-1 kinase, mitogen-activated protein (MAP) kinase, and S6 kinase by hyperosmolality in renal cells. J. Biol.Chem. 269:31296-31301.

46. Chao, T.S., D.A. Foster, U.R. Rapp, and M.R. Rosner. 1994. Differential Raf requirement for activation of mitogen-activated protein kinase by growth factors, phorbol esters, and calcium. J. Biol. Chem. 269:7337-7341.

47. Bogoyevitch, M.A., A.J. Ketterman, and P.H. Sugden. 1995. Cellular stresses differentially activate c-Jun $\mathrm{N}$-terminal protein kinases and extracellular signal-regulated protein kinases in cultures ventricular myocytes. J. Biol. Chem. 270:29710-29717.

48. Schlaepfer, D.D., S.K. Hanks, T. Hunter, and P. van der Geer. 1994. Integrin mediated signal transduction linked to Ras pathway by GRB2 binding to focal adhesion kinase. Nature (Lond.). 372:786-791.

49. Miyamoto, S., H. Teramoto, O.A. Coso, J.S. Gutkind, P.D. Burbelo, S.K. Akiyama, and K.M. Yamada. 1995. Integrin function: molecular hierarchies of cytoskeletal and signaling molecules. J. Cell Biol. 131:791-805. 